\title{
ENSEÑANZA PARA UN CAMBIO CONCEPTUAL: EJEMPLOS DE FUERZA Y DE MOVIMIENTO
}

\author{
HEWSON, P.W.' y BEETH, M. E. ${ }^{2}$ \\ ' Departamento de Programación e Instrucción. Universidad de Wisconsin-Madison. \\ ${ }^{2}$ Departamento de Teoría y Prácticas Educativas. Universidad del Estado de Ohio.
}

\section{SUMMARY}

In this article the possibility of learning as a conceptual change and the way of teaching appropriate for this is discussed. This teaching has to have certain characteristics and ensure that the debate in the classroom is centered on the explicit ideas of the children, the status of which must be discussed and negotiated. To achieve this, the curriculum must give importance to the justification of ideas, the debate must be metacognitive and the role of the professor must be more active and diversified. One example of this way of teaching is presented in the article.

\section{INTRODUCCIÓN}

El papel básico que desempeña el conocimiento real de los estudiantes en cualquier actividad intelectual está hoy ampliamente aceplado, del mismo modo que lo está el descubrimiento de que hay una considerable diversidad en ese conocimiento real entre los estudiantes, de que la diversidad contradice a menudo opiniones generalmente aceptadas y de que gran parte de todo ello parece no responder a la instrucción que reciben. Todo esto proporciona una base para considerar el aprendizaje, no como una simple acumulactón de información, sino como un proceso de cambio conceptual. Ello plantea dos cuestiones: el significado del aprendizaje como cambio conceptual; y lo que una perspectiva de cambio conceptual del aprendizaje nos dice acerca de la enseñanza.

Respecto a la primera cuestión, nosotros utilizamos un modelo del aprendizaje como cambio conceptual propuesto en primer Iugar por Posner, Strike, Hewson y Gertzog (1982) y discutido nuevamente en numerosas ocasiones (Hewson 1981 1982, Hewson y Thorley 1989, Strike y Posner 1985, 1992, Thorley 1990). Los conceptos centrales del modelo son el status y la ecología conceptual. El status que las ideas tienen para la persona que las mantiene es una indicación de su inteligibilidad, de su plausibilidad y de su utilidad. La ecología conceptual incluye otros conocimientos que tiene la persona y que interactúan con esas ideas en el proceso de determinación y de cambio de su status. Estos conceptos se tratan más a fondo en los art́culos citados anteriormente.

Respecto a la segunda cuestión, nosotros consideramos que el modelo del cambio conceptual es un modelo de aprendizaje. Los modelos de aprendizaje no prescriben un tipo de enseñanza. Pueden, sin embargo, utilizarse para analizar críticamente métodos, puesto que se pueden examinar ciertas propuestas de estrategia y de ordenación didácticas para comprobar si facilitan o entorpecen cl aprendizaje diseñado por un determinado modelo. Un proceso de examen como éste pucde conducir a la identificación de pautas generales que sean consistentes con el modelo y pueden servir para eliminar to que resulte inconsistente con él, más que prescribir qué es lo que se necesita. Llamaremos enseñanza para un cambio conceptual a la enseñanza que pretende explícitamente ayudai a los estudiantes a experimentar el aprendizaje del cambio conceptual y a cumplir con las pautas consistentes son el modelo del cambio conceptual.

Diversos autores han discutido las implicaciones de la enseñanza de la investigación conceptual del estudiante y del aprendizaje del cambio conceptual. Esta Iiteraturá, recientemente revisada por Scott, Asoko y Driver(1992), 
ha propuesto una serie de métodos diferentes. En todos ellos aparece la importancia que ellos atribuyen al conocimiento de los estudiantes anterior a la instruccion. Algunos, sin embargo, contemplan este conocimiento como una oportunidad -la base para un futuro aprendizaje-, mientras que otros lo consideran como un problema que hay que superar. Aparece también implícito, en todos los métodos, el papel del profesor como algo esencial, tanto en la elección como en la implantación del plan de estudio. Existen, sin embargo, diferencias en los papeles previstos para los estudiantes, principalmente respecto a la amplitud del control que puedan tener sobre el desarrollo de la instrucción. Las pautas esbozadas más adelante perfilan, en gran parte, este debate todavía vivo.

En este artículo, primero planteamos y discutimos las pautas generales en la enseñanza para una cambio conceptual. A continuación, consideramos varios factores importantes que parecen ser necesarios para cumplix estas pautas en las clases normales; estos factores se refieren al profesor, a los estudiantes y al clima en el aula. Finalmente, ilustramos estas pautas utilizando ejemplos extraídos de las aulas en las que se practica la enseñanza del cambio conceptual.

\section{NORMAS SOBRE LA ENSEÑANZA PARA UN CAMBIO CONCEPTUAL}

Nosotros damos por entendido que una amplia gama de diferentes actividades docentes cumplen estas pautas. Por otro lado, el hecho de prescntarlas en un determinado orden no quiere decir que demos por supuesto un orden secuencial o temporal en la cnseñanza. Analíticamente, estas pautas representan diferentes finalidades que pueden alcanzarse simultáneamente, dependiendo de la actividad elegida en el aula.

\section{Las ideas de los estudiantes tienen que ser una parte explícita del debate en el aula}

En la enseñanza para un cambio conceptual, es necesario que se hagan explícitas las distintas opiniones de los distintos integrantes de la clase sobre el tema. Estas opiniones tienen que ser aportadas tanto por el profesor como por los estudiantes. En el proceso, los implicados serán conscientes de ideas con las que no se habían encontrado antes o no las habian considerado seriamente, y aprenderán a valorarlas. Estas ideas podrian ser o bien las aportadas por otros o bien las que uno mismo ya tenía pero a las que no se había dedicado excesiva atención.

Una parte de esta pauta se fundamenta en las enseñanzas habituales, puesto que los profesores siempre han hecho explícita su opinión al enseñar los objetivos de un tema. Hay, sin embargo, dos diferencias significativas respecto a lo que habitualmente se practica. La primera es la consideración de que las opiniones de los estudiantes desempeñan un importante papel en el aprendizaje. Este paso ha sido defendido ampliamente en la literatura reciente, pero en la mayoría de las aulas no se ha aplicado todavía. Cuando solamente se explicitan las opintones del profesor, algunos estudiantes pueden ser conscientes de que sus propias ideas son distintas de las del profesor, pero no conocerán las opiniones de los demás estudiantes. Tan importante como esto es el hecho de no existir un incentivo para que los estudiantes se tomen en serio sus propias ideas, cosa que puede conducir a la consecuencia no deseada de que los estudiantes devalúen sus propias ideas. Esta pauta apunta a unas prácticas significativamente distintas a lo que acabamos de describir.

La segunda diferencia respecto a lo que habitualmente se practica es que las opiniones de los estudiantes deben considerarse de forma similar a la del profesor. Esto contrasta con la mayoría de las clases de ciencias en las que la presentación de las ideas del libro de texto es básica y casi nunca, si es que se hace alguna vez, se contempla la de los estudiantes. Considerar las opiniones de los estudiantes de esta forma puede parecer sorprendente puesto que la opinión del profesor está, probablemente, más ampliamente desarrollada, más aceptada por la comunidad científica y constituye el objetivo esperado del aprendizaje dentro de Ia enseñanza. La intención de este aspecto consiste en garantizar que los estudiantes elijan entre distintas opiniones sobre la base, no de quien las expone, sino de Ia validez de la cxplicación que cada uno proporciona. AI hacerlo así, deberían llegar a reconocer que la fuente de autoridad de una determinada idea no debería ser la indudable posición de poder del profesor, sino los estándares de convicción aceptados por la disciplina. Una importante consecuencia de este punto es que los estudiantes están constantemente comunicando a los profesores el estado actual de sus ideas, es decir, que la espontaneidad se convierte en un aspecto omnipresente de la actividad en las aulas. Más adelante se consideran otras implicaciones para los profesores, para los estudiantes y para el clima en el aula.

Algunas formas de hacer explícitas las opiniones de todos los estudiantes, al inicio de una nueva sección, son las siguientes:

I. El profesor empieza un tema con una cuestionario que no comporte calificaciones, contestado individuatmente, y que incluya preguntas que tengan un cierto margen de opciones que representen diversas opiniones corrientes. Tras el cuestionario, el profesor describe la muestra de las respuestas de la clase y pide a los estudiantes que expliquen sus opciones (Minstrell 1982).

2. El profesor of rece algunas demostraciones para introducir el contexto del trabajo. Los estudiantes trabajan individualmente mediante actividades espontáneas, discuten sus respuestas por parejas, preparan, en grupos de cuatro, carteles que resuman sus conclusiones y presentan esos carteles a la clase (CIIS 1987).

3. Los estudiantes definen lo que significa para ellos un término o un concepto antes de la clase lectiva. 
(Hennessey 1991). Essta técnica viene ilustrada más adelante.

\section{El status de las ideas tiene que ser discutido y negociado}

La enseñanza para un cambio conceptual debe conducir a los estudiantes a considerar diferentes opiniones, conduciéndoles a la necesidad de elegir a través de la información. Sus posibles elecciones pueden ser seguir prefiriendo sus previas opiniones, aceptar más de una opinión, o preferir una opinión distinta, a expensas de su propia opinión previa. Vale la pena señalar que adoptar cualquiera de estas opciones no exige la desaparición de las opiniones rechazadas. En el proceso de elección, los estudiantes pueden encontrar que algunas opiniones les parecen más aceptables y otras menos. En otras palabras, el status de estas opiniones cambia, subiendo en algunos casos y descendiendo en otros. A veces, estos cambios pucden ser interdependientes, por ejemplo, cuando un estudiante cambia su parecer acerca de dos opiniones que le resultan mutuamente contradictorias.

Hay que tener en cuenta que cuando se elige, la elección no sólo depende de las opciones disponibles sino también de las consideraciones que intervienen en la elección. Estas consideraciones y el conocimiento necesario para aplicarlas son parte de la ecología conceptual de cada persona. Puesto que to más probable es que exista una considerable variedad entre ecologías conceptuales, es probable que las diferentes personas elijan de forma distinta. El concepto de elección está ligado a la pauta, puesto que una persona elige una opción frente a otra debido a que, desde su punto de vista, el status de una opción es más alto que el status de la otra. Dicho de otro modo, las personas utilizan su ecología conceptual para determinar los status; éstos están, probablemente, implícitos, y solamente se expresan en el lenguaje de status en ocasiones especiales, aunque, no obstante, son básicos. Por ejemplo, un esurdiante rechaza la afirmación de que la mesa ejerce una fuerza hacia arriba sobre el libro para sostenerlo debido a que no puede imaginarse cómo auna mesa muerta puede saber cuánto tiene que empujar hacia arriba» (Hennessey 1991). Esta opinión tiene un status bajo para él o ella; sabe lo que quiere decir, pero no acaba de creérselo. En otras palabras, la necesidad de que sus explicaciones proporcionen mecanismos causales aceptables es un componente importante de su ecología conceptual. Otro estudiante acepta esta opinión explicando cl estado de reposo del libro mediante la compensación de fuerzas, una explicación que aparece en otros cjemplos. En otras palabras, su compromiso epistemológico-ejemplos similares exigen explicaciones similares-constituye un instrumento para elevar el status de su opinión; es el criterio que utiliza para hacer su elección. La pauta, por tanto, sugiere que el profesor tiene que ser consciente de la importancia de ambos status en las opiniones de los estudiantes y de los componentes de sus ecologías conceptuales, e indica que necesita incluir explícitamente ambos status y consideraciones ecológicas en la enseñanza en el aula (Hewson y Thorley 1989. Hennessey 1991).
Las actividades enfocadas a elevar el status de ideas particulares deberían, por consiguiente, formar parte de la eriseñanza para un cambio conceptual. Desde este aspecto, hay mucho en común con la enseñanza habitual. Estas actividades pueden llevarse a cabo para presentar y desarrollar las ideas, para proporcionar ejcmplos, para aplicarla en otras circunstancias, para ofrecer distintas maneras de pensar, para enlazar con otras ideas, etc. Las actividades enfocadas a disminuir el status de otras ideas también deberían formar parte de la enseñanza para un cambio conceptual. Éstas pueden lłevarse a cabo para explorar sus implicaciones inaceptables, para considerar experiencias que son incapaces de explicar, para encontrar formas de pensar que apuntan a sus imperfecciones. El hecho de que una actividad encaminada a disminuir el status funcione o no, para un estudiante en particular, exige que el estudiante capte la imperfección de la idea; un problema habitual consiste en que los profesores suponen, a menudo y erróneamente, que la discrepancia resulta tan obvia para el estudiante como para ellos mismos. Es importante señalar que las actividade; encaminadas a elevar o a disminuir el status pueden realizarse simultáneamente.

En muchas aulas, Ia labor de tos profesores no conduce a la consideración de diferentes opiniones. Fn tales circunstancias, la posible necesidad de disminuir el status de sus ideas previas y las acciones para facilitar tal disminución no constituirán una parte explícita del trabajo en el aula. Si los profesores no ofrecen la oportunidad de que esto ocurra, no podrá considerarse, bajo nuestro punto de vista, que están enseñando para un cambio conceptual. Dicho lo cual, es importante señalar que no es necesario que se realice la enseñanza para un cambio conceptual para que el aprendizaje del cambio conceptual tenga lugar: parece más apropiado considerar la enseñanza para un cambio conceptual como un catalizador más que como un agente causal del aprendizaje del cambio conceptual.

\section{La justifícación de las ideas tiene que ser un componente explícito del plan de estudio}

En el aprendizaje del cambio conceptuai, los estudiantes determinan el status que una idea tiene para ellos. Por lo tanto, primero tienen que saber lo que es esa idea, es decir, saber si es inteligible, y después decidir si encuentran la idea plausible o no, o si les resulta útil. Con el fin de proporcionar una justificación para tal decisión sobre el status, los estudiantes mantendrán uno o más criterios, siendo éstos parte de los componentes importantes de sus ecologías conceptuales en las que van a situar la idea considerada. Vamos a considerar las bases para algunas justificaciones de plausibilidad; las bases para la utilidad serían similares.

Llegar a considerar una idea como plausible se puede conscguir creyendo que es cierta o considerándola consistente con otros conocimientos plausibles. Al considerar la plausibilidad, aparecen al menos dos categorías relevintes de componentes de la ecología conceptual. La primera es la creencia metafísica: La que viene dada y no 
puede probarse, la naturaleza misma de la cosa. Si una idea que está siendo considerada por un estudiante contradijese una de sus creencias metafísicas, ello se convertiría en una base para decidir que la idea no es plausible. Un estudiante que no acepta que una mesa puede ejercer un fuerza para sostener un libro que está sobre ella puede que no lo acepte debido a su creencia metafísica acerca de los objetos inanimados: ¿cómo pueden «saber cuánto tienen que empujar»?

La segunda categoría es un compromiso epistemológico: el criterio que utiliza una persona para decidir si un conocimiento está justificado y es aceptable o no. Tal compromiso se refiere a la consistencia. Si una persona cree que una idea es consistente, de forma significativa, con otra idea aceptable, ello se convierte en una base para decidir que la primera idea es plausible. Considerar que un libro en reposo sobre una mesa es similar a un libro en reposo sostenido con una mano en el aire puede llevar a decidir que la consistencia exige una explicación similar: gravedad contrarrestada por una fuerza hacia arriba ejercida sin duda por la mano, en un caso, y por la mesa en el otro. Otro de los compromisos se refiere a la causalidad. Una idea puede considerarse como plausible si existe una mecanismo causal que la explique. Un ejemplo sería el de ser capaz de imaginar que una mesa puede estar compuesta de partículas con fuerzas mutuas que actúan a modo dc muelles y que serian empujadas hacia atrás cuando se colocara un objeto sobre ellas. Esta sería una manera eficaz para que una persona aceptase que la mesa puede ejercer una fuerza hacia arriba.

La determinación de la plausibilidad y de la utilidad (tanto si se utiliza como si no se utiliza el lenguaje del cambio conceptual) no constituye un componente significativo en la mayoría de las aulas y, sin embargo, es una parte esencial del aprendizaje del cambio conceptual. Esta pauta se refiere a la necesidad de una consideración explícita de las bases para la determinación del status. Si bien esta pauta cstá implícita en la anterior (la necesidad de una consideración explícita del status), se incluye aquí para destacar el papel esencial de los componentes de la ecología conceptual de una persona, en el aprendizaje del cambio conceptual.

\section{El debate en el aula tiene que ser explícitamente metacognitivo}

La enseñanza para un cambio conceptual es explícitamente metacognitiva. El concepto de metaconocimiento se refiere al «conocimiento acerca de los procesos y resultados cognitivos de uno mismon (Flavell 1976). Los aspectos del proceso cognitivo del metaconocimiento ya han sido destacados en estudios de otras áreas, por ejemplo, en la lectura, en la cual hacen referencia al conocimiento y control de los factores que afectan a la actividad de aprendizaje, tal como el conocimiento de sí mismo en tanto que alumno, las exigencias de la tarea de aprendizaje y las estrategias utilizadas para aprender (Palincsar y Ransom 1988). Si bien estos aspectos son importantes en cualquier forma de aprendizaje, el cono- cimiento sobre los resultados cognitivos de uno mismo es particularmente importante en la enseñanza para un cambio conceptual. Thorley (1990) aplicó una distinción muy útil al utilizar los términos «metacognitivo» y «metaconceptual», para referirse a la reflexión sobre el proceso cognitivo y al «contenido de las propias concepciones», respectivamente. En otras palabras, cuando los estudiantes no solamente piensan en sus ideas sobre el fenómeno, sino que piensan acerca de esas ideas, están siendo metaconceptuales.

¿Por qué el metaconocimiento, en general, y la metaconcepción, en particular, constituyen un hito en la enseñanza del cambio conceptual? Cuando los profesores exponen en clase diferentes explicaciones acerca de un fenómeno en particular, o acerca de un conjunto de fenómenos, están de hecho presentando las explicaciones como si esas mismas explicaciones fueran, a su vez, objetos de conocimiento; es decir, están siendo metaconceptuales. Cuando los estudiantes comentan, comparan y contrastan estas explicaciones, cuando consideran los argumentos que apoyan o contradicen una u otra explicación, y eligen una de las posibles explicaciones, están realizando actividades metaconceptuales.

Debería quedar claro que la metaconcepción reptesenta otra manera de describir la intención de las dos pautas anteriores y que, por tanto, está implícita en ellas. Fl hacerlo explícito sirve, sin embargo, para otorgar al meta-conocimiento una identidad que, desde nuestro punto de vista, proporciona una importante perspectiva en la enscñanza para un cambio conceptual.

\section{FACTQRES QUE SIRVEN DE BASE A LA ENSENANZA PARA UN CAMBIO CON- CEPTUAL}

\section{EI profeser}

En la enseñanza para un cambio conceptual, los profesores tienen diversos papeles que desempeñar. Un papel es el de «allanador» responsable de establecer en el aula el clima que esbozaremos más adelante, con el fín de «allanar» al estudiante el camino hacia el aprendizaje. Esto conlleva establecer los contextos apropiados para las actividades en el aula, planteando problemas que tengan relevancia y sentido para los estudiantes, explorando las distintas ideas sin temor a aquéllos que las tienen, encontrando las formas de ayudar a los estudiantes a sentirse satisfechos con sus propias ideas y proponiendo tareas en las cuales los estudiantes aptiquen las ideas recién adquiridas. También exige que el profesor o la profesora establezca las reglas de base que hayan de regir todos los aspectos de la interacción en el aula, que las discuta explícitamente en la clase y que las aplique de forma consistente.

Un segundo papel consiste en ser un participante activo en la clase. Se presenta una disyuntiva importante en este punto. Por un lado, es muy fácil que la voz del profesor 
sca la más potente de la clase; en muchos casos, la única. En el otro extremo, el profesor podría utilizar principios de aprendizaje por descubrimiento, dando por supuesto que toda la información proviene de la experiencia, es dccir, que el profesor no se haga oír para transmitir contenidos. Es necesario conseguir un equilibrio entre estas dos posiciones; es tan importante escuchar las ideas del profesor como las de los estudiantes. Es, también, importante que los estudiantes se stentan tan libres de rechazar las ideas del profesor como las de sus propios compañeros.

Para desempeñar estos papeles con éxito se necesitan condiciones importantes en el profesor. Las secciones previas ya señalaban algunas: un respeto por y un conocimiento de los alumnos y de sus ideas, una comprensión del desarrollo histórico de los conceptos en relación con las vías de aprendizaje de los estudiantes, y un amplio repertorio de estrategias apropiadas de enseñanza y de materiales de soporte. Implícitas en todas estas condiciones están las concepciones del profesor sobre la naturaleza del aprendizaje, de la enseñanza y de la ciencia que sirven de apoyo en la enseñanza para un cambio conceptual. Estas ya han sido expuestas en otro lugar (Hewson y Hewson 1988).

\section{El alumno}

Los estudiantes tienen papeles específicos en las aulas en las que los profesores tengan como objetivo la enseñanza para un cambio conceptual. Es necesario que se conviertan en alumnos convencidos de que el objetivo del aprendizaje tiene que ser entender el tema que se está considerando $y$, consiguientemente, hacerlo suyo. Por tanto, necesitan aceptar la responsabilidad de su propio aprendizaje, hacerse conscientes de sus propias ideas y de sus propias razones para aceptarlas, confiar en su propio pensamiento y justificar sus conclusiones utilizando argumentos sensatos. Al hacerlo, sin embargo, tienen que reconocer que puede haber diferentes puntos de vista sobre del mismo suceso y que esos puntos de vista deber ser respetados y evaluados en relación con sus propias ideas. Cuando se expresan distintos puntos de visia, los estudiantes deben escucharlos y entenderlos, y regociar significados comunes. Finalmente, deben estar dispuestos, a la luz de la comparación y del contraste de pareceres, a cambiar sus ideas cuando otra sea más viable.

\section{EI clima en el aula}

El clima en el aula en la que se está enseñando con el objetivo de un cambio conceptual presenta trazos significativos. Tanto el profesor como los estudiantes ticnen que respetar las ideas de los demás de la clase y escucharlas atentamente, incluso si no están de acuerdo con cllas. Es esencial para los participantes que sean capaces de expresar sus ideas abiertamente, sin miedo a la sanción o al ridículo, y que sean capaces de expresar su desacuerdo con las ideas de los demás y de pedir aclaraciones sobre las explicaciones de los demás. Otra dimen- sión en esta materia es la necesidad de separar persona e idea: ser capaz de criticar una idea al mismo tiempo que aceptar a la persona. Una estrategia para conseguirlo sería la de ocultar la identidad de la fuente de la idea, por ejemplo, presentando las ideas por grupos, recogiendo las ideas por escrito de forma anónima o presentando las ideas unos por otros. Tal como dijimos anteriormente, es el profesor el que debe cstablecer el clima en el aula.

Además, tiene que haber una aceptación común de que el objetivo del debate es la consecución de significados compartidos acerca de los temas que se discuten. Puesto que hay que reconocer la diversidad de las ideas iniciales en el aula, para llegar a este objetivo es necesaria la voluntad de hacer un esfuerzo por entender los puntos de vista de los demás, por negociar y por llegar a compromisos. Se trata de un proceso que requiere tiempo y que puede ser fácilmente subvertido mediante ciertas tácticas, como la conclusión prematura del debate. En este proceso por alcanzar el consenso está implícita su naturaleza social. Un acuerdo de consenso es el resultado de la tolalidad del grupo. Es más que la suma de las ideas particulares que cada persona mantenía al iniciarse la discusión, es decir, que el acuerdo se establece socialmente por el grupo.

Finalmente, es necesario adoptar los significados negociados, no porque el profesor lo diga, sino porque tienen sentido para los participantes. En otras palabras, la base de la aceptación debe ser la racionalidad del tema en cuestión, más que la autoridad de la fuente del significado aceptado, sea un libro de texto, el profesor o un estudiante en particular. Un aspecto importante para conseguir este fin es la consideración imparcial de las ideas esbozadas en las pautas anteriores.

Estos trazos tienen que ser operativos en todo momento en el aula, es decir, tanto durante la presentación de las diferentes ideas como durante las actividades de cambio de status. Sin embargo, no constituyen una práctica habitual en muchas aulas. Con frecuencia, solamente se presenta una opinión a debate, la del profesor. Se supone implícitamente que las ideas de los estudiantes son copias (probablemente imperfectas) de las del profesor y que, por tanto, no tienen ninguna implicación. Se transmite la opinión del profesor a los estudiantes y la única negociación se centra en si los estudiantes han recibido el mensaje o no lo han recibido, independientemente de si este mensaje tiene o no tiene sentido para ellos.

\section{EJEMPLOS DE ENSEÑNANA PARA UN CAMBIO CONCEPTUAL}

Vamos a ilustrar las pautas y factores discutidos hasta ahora mediante dos ejemplos extraídos de una clase e intensamente estudiados por uno de nosotros (Beeth 1993). Estos ejemplos no pretenden ser afirmaciones definitivas acerca de la enseñanza para un cambio conceptual -tal como hemos indicado anteriormente, otros 
métodos de enseñanza pueden también cumplir las mismas pautas- sino como una elaboración de posibilidades que contribuye al debate. Antes de discutir los dos ejemplos, presentamos el contexto del que se han extraifdo.

\section{El contexto}

Los ejemplos provienen de un estudio realizado durante un año de enseñanza en un aula de quinto grado (Beeth 1993). Había 13 alumnos (8 chicos y 5 chicas, con edades entre 10 y 11 años) que vivían en una comunidad mayoritariamente blanca y de clase modia, de diez mil habitantes, dentro de un área bien comunicada con la cercana capital del estado. Todos tos alumnos que participaron en el estudio tenían las características típicas de los alumnos de quinto grado, sin que ninguno necesitara ayudas especiales de ningún tipo, por lo que se refiere a sus capacidades intelectuales. Tenían 3 clases de ciencias por semana.

La profesora, I fermana M. Gertrude Hennessey, enseñaba ciencias a los grados del 1 al 6 y era vice-directora de Ia escucla, una escuela elemental pequeña de ámbito parroquial, con un solo grupo de alumnos por grado. En otras palabras, todos los alumnos de este estudio, excepto aquéllos que se incorporaron a la cscucla en los grados superiores, habían estudiado ciencias con la Hermana Gertrude durante 5 años consecutivos. El programa de ciencias de la escuela, que estaba bajo su supervisión, estaba coherentemente estructurado para los 6 grados. Una muestra de ello lo constituyen los objetivos de aprendizaje (Learning Goals) que se introdujeron en el primer grado y que fueron incorporados de forma consistente a lo largo del resto de los grados (Tabla I).

Tabla I

Los objctivos de aprendizaje del profesor.

1. ¿Puedes expresur tus propias ideas?

2. ¿Puedes hablar accrca de por qué te sientes atraído por Ius ideas (e) "qué» y también el "porqué»)?

3. ¿Son consistchtes us idcas?

4. ¿Eras consciente de las limitaciones de tus ideas y de la posibilidad de que tengas que cambiarlas?

5. ¿Pucdes intentar explicar tus ideas utilizando modclos físicos?

6. ¿Puedes explicar la diferencia entre entender una idca y creer en una idea?

7. ¿Puedes aplicar los conceptos de inteligibilidad (I) y plausibilidad (P) ly utilidad (U)] a tus propias ideas?

\section{Nota}

Los objetivos 1 y 2 se presentan a los estudiantes cuando empiezan las clases con la Hermana Gertrude como alumnos de primer grado (edades de 5 a 6 años) El resto de los objelivos se van añadiendo hasta quc, hacio cuarto grado (edades de 9 a 10 años), se ha cubierto la lista completa.

Si bien la Hermana Gertrude eligió los temas, cl enfoque detallado de cada clase se basaba en el contenido de las ideas de los alumnos sobre el tema y en las razones por las cuales sostenían tales ideas. De esta forma se garantizaba que la exposición, la consideración de statiss y el metaconocimiento fueran una parte central del programa. La Hermana Gertrude inició el año escolar de $5^{\circ}$ grado con unas sesiones acerca de la inteligibilidad y la plausibilidad, términos extraídos del modelo del cambio conceptual. La información presentada en estas sesiones serviría a los alumnos para el resto de su educación en ciencias. Los temas de ciencias tratados durante el año comprendian un repaso de la naturaleza de las partículas materiales (contenido visto en $4^{\circ}$ grado) y un punto dedicado a fuerza y movimiento, tema nuevo para los alumnos.

Las actividades educativas diseñadas para familiarizar a los alumnos con el lenguaje del cambio conceptual llevó a una comprensión general de cómo se iban a utilizar estos términos en la clase. Más adelante, cn cste artículo, presentamos una descripción del método que condujo a la definición de consenso sobre el concepto de inteligibilidad, y damos ejemplos de algunos segmentos del debate que contienen referencias a dichas definiciones de consenso. No exageramos si decimos que las ideas de los estudiantes fueron el centro constante de atención en el programa de esta clase. Una de las preguntas habituales de la Hermana Gertrude, tras la exposición de una idea era: «¿Qué quieres decir cuando dices [la idea]?» Esta pregunta, aparentemente simple, se repetía como respuesta a casi todos los planteamientos hechos por los alumnos y permitía que surgiera de ellos el razonamiento y las creencias en los que se basaban sus ideas. Exigia, además, que el alumno se lanzara a una actividad metacognitiva que, eventualmente, iba a revelar cl status de una idea y los componentes de la ecología conceptual dentro de los cuales esta idea tenía sentido para él o para ella.

Otra dccisión programática tomada por la Hermana Gertrude y que contrasta con gran parte de la enseñanza tradicional de lás ciencias era que ella nunca introducía las ideas actuales sobre el fenómeno científico, o sobre ningún tema de la materia, hasta el momento en que los estudiantes ya «no pudieran seguir avanzando con sus propias ideas» (comunicación personal). Si bien ella presentaba a los alumnos ciertos precedentes históricos sobre las ideas científicas, nunca presentaba las ideas científicas contemporáncas como un cstándar frente al cual hubiera que juzgar cl resto de las ideas. Su método consistía en insinuar que «hay personas, a las que llamamos científicos, que tienen ciertas ideas acerca de [las fuerzasl, pero sus ideas también han evolucionado con el ticmpom.

\section{Desarrollo del consenso sobre inteligibilidad}

El primer cjemplo describe el tratamiento de la Hermana Gertrude de una unidad didáctica completa. Se trata de proporcionar una visión del conjunto general de las actividades educativas mediante las cuales los alumnos manifestaron su conocimiento sobre el tema y llegaron a un consenso sobre la comprensión del mismo. Éste fue el 
ciclo general educativo vivido por estos estudiantes, independientemente de que estuvieran estudiando un tema de ciencias o el lenguaje del cambio conceptual. El objetivo de la unidad didáctica descrita aquí consistía en desarrollar la comprensión del término «inteligible».

El proceso se desarrolló a través de varias etapas en las que los alumnos trabajaron solos, en pequeños grupos 0 todos juntos en discusiones colectivas de la clase. Los estudiantes empezaron escribiendo de forma individual una definición del término «inteligible» que tuviera sentido para ellos. A continuación, trabajó conjuntamente toda la clase para obtener una lista de términos que significaran «inteligible», entre los cuales aparecieron los siguientes descriptores:

entender, hábil, claro, estar de acuerdo, pensar, acerca de, saber cosas, compartir ideas, resolver, brillante, listo, sobresaliente, sabio, perspicaz, capaz de ser comprendido, y comprensible.

Después, se formaron grupos de tres a cinco alumnos para discutir lo que para ellos significaba «inteligible» y para alcanzar un consenso sobre el significado. El sistema de alternar la discusión en pequeños grupos con la discusión de toda la clase se fue repitiendo en sucesivas sesiones conforme los alumnos iban perfilando sus pensamientos acerca de lo que resultaba o no resultaba apropiado como definición de «inteligible». En las sesiones de toda la clase, la Hermana Gertrude preguntaba de forma reiterada a los alumnos: « $i$ Por qué crees que [tu respuesta] es una buena definición de inteligible?». O bien: «¿Puedes darme alguna razón de por qué crees que [tu respuesta] es una buena defínición de inteligible?». En un momento dado, durante el proceso, se les pidió a los alumnos que escribieran una definición de «inteligible». Jack y Pete, por ejemplo, escribieron:

Cuando uno explica su idea, las palabras y las frases tienen que tener sentido. Uno puede hablar, dibujar y escribir sobre su idea. Comprensible y tener sentido tienen básicamente el mismo significado. Uno puede hablar acerca de su idea con modelos. Cuando uno explica su idea tiene que quedar clara para que la gente entienda y darle algún sentido. Uno puede usar modelos y analogias para representar sus ideas. La idea tiene que ser consistente en la misma situación. Uno puede utilizar sus experiencias para construir sus ideas. Antes de construir sus ideas uno tiene que tener su estructuración previa o su comprensión básica sobre ellas. Si uno explica su idea utilizando modelos, éstos tienen que ser claros, o tener sentido para los demás. La explicación tiene que ser comprensible para los demás.

A continuación, la profesora presentó a los estudiantes el «conocimiento educativo aceptado» en forma de definiciones del término «inteligible» extraf́das de la literatura sobre el cambio conceptual. Los estudiantes leyeron estos extractos y utilizaron la definición de «inteligilidad" que ellos habían elaborado para comentar si los extractos les resultaban inteligibles o no. Los estudiantes fueron capaces de leer los extractos detenténdose solamente cuando Ios autores utilizaban palabras desco- nocidas para ellos, tales como epistemología u ontológico. Sin embargo, los alumnos afirmaron de forma general que, si hubieran sabido lo que querían decir palabras como epistemología u ontológico, hubieran podido entender lo que el autor decía. La definición que esta clase no tavo dificultad en entender, y la que encontró más próxima a sus propias definiciones, había sido escrita por la clase de sexto grado del año anterior.

A los estudiantes les faltaba todavía alcanzar el consenso para una definición de grupo de inteligibilidad. La Hermana Gertrude utilizó los carteles en los que se habían escrito los pensamientos de los estudiantes sobre «inteligible», para hacer que seleccionaran formulaciones acerca de las cuales «todos estuvieran de acuerdo» en que representaban su idea de «inteligible» y formulaciones sobre las cuales «algunos estuvieran de acuerdo» en que representaban su idea de «inteligible». Las formulaciones que eran suficientemente similares para que todos estuvieran de acuerdo se copiaron sobre un gran cartel titulado "Definición de Grupo del Término Inteligible» (Tabła II), lo que constituyó la actividad de clausura de esta unidad didáctica.

\section{Tabla II}

Definición de grupo para el término «inteligible».

- E. término inteligible puede utilizarse para describir:

1) ris propias ideas;

2) las ideas de otra-otro / las ideas de los demás

- Cuando digo que una idea me resulta inteligible quicre decir que creo que entiendo la idea.

- Hay dos mancras de ayudarme a decidir si entiendo una idea:

1) Tengo que decidir si entiendo de qué iratan las palabras, las frases y Jas ideas o lo que quieren decir;

2) Tengo que decidir si las palabras y las ideas que hay detrás de las palabras tienen sentido para mí.

- Cuando decido que entiendo una idea tengo que ser capaz de:

1) encontrar la manera de representar mis ideas para los demás: mediante dibujos o ilustraciones;

2) hablar sobre mis ideas o explicarlas a los dernás;

3) utilizar analogias o dar ejemplos para explicar o hacer que mis ideas queden claras para los demás.

Hay varios puntos interrelacionados que merecen la pena destacarse respecto al proceso que acabamos de presentar. El primero es que, a lo largo del desarrollo de este punto del programa, la clase fue proporcionando a la profesora informacion, constantemente actualizada, solyre sus ideas. Los alumnos lo hicieron de forma individualizada al inicio de las sesiones, reiteradamente durante las discusiones en pequeños grupos o con la clase entera, y en el momento de la formulación de la definición de grupo. De este modo, entre otras cosas, este proceso podría considerarse como una exposición continuada.

Segundo, la clase alcanzó su decisión de consenso a través de una serie de aproximaciones sucesivas. Ello 
permitió detectar confusiones, llenar vacíos de comprensión y desarrollar razonamientos que apoyaran una idea frente a otras. Al hacerlo, la clase consideró un cierto número de posibitidades diferentes, algunas de las cuales fueron rechazadas y otras fueron aceptadas. En otras palabras, los alumnos fueron discutiendo sobre los diferentes status de las diferentes opciones. Este hecho, a nuestro entender, apoya la consideración del aprendizaje no como algo simple y unidireccional, sino como algo complejo y cíclico y que requiere una constante revisión de todos los aspectos de una idea central.

Además, la profesora desempeñó varios papeles diferentes durante el desarrollo. Ella decidió la idea central sobre la que trabajar, el tipo y la secuencia de las tareas educativas y el momento en que había que introducir ideas nuevas. La profesora indujo constantemente a los estudiantes a razonar lo que estaban diciendo y decidió cuándo había que pasar a una nueva idea. Todos estos papeles de control son básicos. Pero, en otros aspectos, ella cedió la iniciativa a los alumnos: escuchó atenta y constantemente lo que decían y no impuso a la clase sus ideas acerca de la inteligibilidad. Una ventaja de observar constantemente la comprensión de los estudiantes era que proporcionaba una firme garantía contra una conclusión prematura de la discusión, además de que servía para demostrar que la fuente de autoridad sobre una idea tiene que ser la argumentación razonada más que la posición preeminente del profesor en la clase.

Los estudiantes tuvieron papeles muy activos en estas sesiones. Estuvieron constantementc atentos a escuchar a Ios demás, pensando en sus propias ideas y resumiendo conclusiones. Todo esto es indicativo del punto hasta el cual habian asumido la responsabilidad de su propio aprendizaje y destaca también la importancia de la naturaleza social de la elaboración de una decisión de consenso.

El resultado final -..la definición de grupo de la inteligibilidad-proporciona una prueba del potencial que representa este tipo de enseñanza. La definición de grupo es mucho más que una definición verbal. Contiene varias partes: saber cómo deciúr si una idea es inteligible, saber lo que puede hacerse con un término que resulta inteligible y saber que «inteligible» puede utilizarse para referirse a pensamientos propios o ajenos.

\section{Negociación del status: Fuerzas que actúan en un descenso en paracaídas}

En el siguiente intercambio, un estudiante, Don, present 6 su explicación sobre las fuerzas que actúan sobre un paracaidas de juguete que descicnde desde el techo hasta el suelo. Don había marcado un punto en la pizarra para representar el paracaídas y dos flechas de magnitudes iguales y opuestas que simbolizaban las fuerzas que actuaban sobre el paracaídas. Para Don, el paracaídas se movía según una línea recta a una velocidad estable, con una marcha uniforme -un físico lo llamaria velocidad constante-. Algunos de sus compañeros de clase consideraron el movimiento del paracaídas como una aceleración, describiéndolo como si fuera cada vez más despa- cio; otros no creían que pudiera moverse si las fuerzas fueran iguales y opuestas, es decir, compensadas.

Don: -Bueno, fui yo quien hizo el paracaidas y creo que hay [dos] fuerzas iguales $y$, puesto que va como en una linea bastante recta y con velocidad estable y esas dos flechas son... ésta es la gravedad y esta otra es la fricción.

Esta declaración fue seguida de una muy rápida secuencia de preguntas de una serie de alumnos que intentaban entender por qué Don creía que esta idea era plausible. Todos los alumnos que hablaron en ese momento se mostraron muy seguros de sí mismos al formular sus preguntas y habia, en la clase, la sensación de que la idea de Don iba a ser rechazada sobre la base de que el paracaídas iba cambiando de velocidad y, por consiguicnte, tenía que haber fuerzas no compensadas que actuaran sobre él.

Kitt: -Bueno, ¿por qué has puesto flechas iguales? Yo no creo que se moviera si tuvieran flechas iguales

Don: -Bien si [una flecha [uera] menor harian que fuera más de prisa

Kitt: - Bien...

Ese «bien...» de Kitt indica claramente que ella creía que el paracaídas iba cada vez más de prisa. Un poco después, Kirsty (cn adelante abreviado a Kirs) continuó la conversación:

Kirs: -Bueno, ¿puedes repetir por qué crees que son flechas iguales?

Don: -Porque va a velocidad estable y creo que si fueran desiguales, entonces [el paracaídas] iria cada vez. más de prisa o más despacio.

La niña, ayudada por la profesora, introdujo otro ejemplo a modo de comparación.

Kirs: -No estoy segura de que esto tenga mucho que ver con esto pero si el paracaídas estuviera parado, icomo serían las flechas? ¿No crees que no está parado?

Don: -.Bien... [no está\} parado.

Kirs: - No, yo quiero decir si estaba parado.

Profesora: -Ella sólo dice en tu cabeza... que imagines que esto esiá parado. ¿Cómo lo indicarías en el dibujo?

Don: -Probablemente [no cambiaria] nada.

Profesora: -No [cambiarfas] nada?

Ellen daba una interpretación distinta de la idea de Don.

Ellen: -Entonces se quedaria flotando.

Don: - ¿Parado] en el suelo? 
Ellen: -No. En el aire.

Stu: -Bueno como si estuviera en alguna parte... si estuviera en alguna parte [en el aire].

Kirsty siguió con su comparación del paracaídas y un objeto en reposo.

Kirs: ${ }_{-1}$ Siát ¿ cómo lo indicarías si estuviera parado? Piensa si estuviera encima de esta mesa, en una parada, piensa si estuviera ahi encima icomo lo indicarías parado? ¿No crees que es eso lo que es estar parado [dos flechas iguales y opuestas]?

Don:-Bueno la gravedad [es una fuerza] y la mesa es una fuerza.

Kitt volvió a intervenir en la conversación para extraer otra conclusión que sería inmediatamente confirmadá por Rob.

Kitt: -De esta forma sería exactamente así [dos flechas iguales y opuestas]... de acuerdo ¿no iría a una velocidad estable?

Rob: - ¡ Claro! [Dijo enfáticamente].

Entonces, Don planteó explícitamente la coincidencia que él veía entre objetos en reposo y objetos moviéndose a una velocidad estable.

\section{Don: -Parado es también a una velocidad estable.}

Stu: -Luego, btienes dos [ideas] para lo mismo como los mismos conjuntos de flechas?

Don: -Si una cosa está parada sigue yendo en línea recta a una velocidad estable.

La discusión dejó a todos los presentes con la sensación de que había ocurrido algo importante. Los alumnos la cmpezaron con mucha confianza en sus concepciones acerca de las fuerzas que actúan sobre objetos en reposo y en movimiento, tanto en las preguntas que se hacían entre ellos como en la creencias que mantenían sobre lo que significaban las flechas iguales y opuestas. Diagnosticaron la inteligibilidad y la plausibilidad de la idea de Don y preguntaron acerca de las razones que había detrás de esta idea a la luz del compromiso que mantenía cada uno con la consistencia y la capacidad de generalización con las cuales podían argumentar a favor o en contra del punto de vista de Don.

Este ejemplo ilustra varias materias distintas. Un cierto número dé alumnos habló explícitamente de sus ideas. La discusión constituyó, entre otras cosas, un excelente ejercicio expositivo.

Además, la conversación de la clase entrañó una negociación de status. Los alumnos probaron diferentes métodos para cuestionar la plausibilidad de la explicacion de Don: Kitt se preguntó si el paracaídas ibáa a una velocidad estable, Kirsty le advirtió a Don que estaba utilizando la misma explicación (fuerzas compensadas) para el paracaídas en movimiento que la clase había dado para los objetos en reposo, y Ellen se preguntaba si el paracaídas no se quedaría flotando en reposo en medio del aire al tener dos fuerzas iguales actuando sobre él. Algunos estudiantes vieron la plausibilidad de la posición de Don cuando se dieron cuenta de lo que Don había estado argumentando desde el principio, a saber, que un objeto en movimiento a una velocidad constante se explica, en términos de fuerzas, igual que un objeto en reposo. Aunque Don precisó esta conexión en sus comentarios finales, algunos de sus compañeros de clase siguieron sin considerar que uen reposo" y «a velocidad estable» respondieran al mismo conjunto de fuerzas, es decir, no aceptaron la explicación de Don como plausible. Parece claro que esa discusión acerca del status de la explicación de Don fue básica para la agenda de clase.

Tercero, un componente esencial de la explicación de Don fue su utilización de una argumentación de consistencia. Una vez que se dio cuenta de la similitud esencial entre el paracaídas descendiendo y el libro sobre la mesa (ningtuno de los dos aumentaba o disminuía su velocidad), argumentó mediante la misma explicación (fuerzas iguales y opuestas) diciendo: la consistencia requicre que se dé la misma explicación frente al mismo efecto. Nosotros interpretamos este hecho como una prueba del compromiso epistemológico de Don con la consistencia.

Cuarto, negociar el status es, entre otras cosas, un acto de metaconocimiento. Una idea concreta -la explicación de Don en este caso- fue, metafóricamente hablando, puesta sobre la mesa. Kitt, Kirsty y Ellen comentaron y criticaron las diferentes implicaciones que para ellas tenía dicha idea: pensaban en la idea de Don, no mediante esa idea. Esto, a nuestro entender, es básico para lo que significa ser metacognitivo. En otras palabras, este segmento proporciona un excelente ejemplo del tipo de debate metaconceptual que parece ser necesario en la enseñanza para un cambio conceptual.

Por último, la discusión comportó una imagen de ambiente serio, reflexivo e intelectual en la clase. Los extudiantes tuvieron con Don Ia deferencia de escuchar atentamente su explicacion. Si no estaban de acuerdo con él, centraban su atención en razonamientos que contradijesen su explicación, en lugar de limitarse a rechazarla o a atacar a Don con una argumentación ad hominem. Le plantearon a Don preguntas que fueron, normalmente, sobre la plausibilidad de su idea: razones y justificaciones que apoyasen su idea. Don respondió a las preguntas con argumentos razonados, con una argunento de consistencia, de cómo había Ilegado a generalizar su concepción del movimiento de los objetos y de I $i$ s fuerzas que actuaban sobre esos objetos. Las afirmaciones de los estudiantes acerca del contenido científico evidenciaron que podían, con sus capacidades metacog. nitivas demostradas en este caso, participar en un debate que facilitara su aprendizaje de conceptos científicos. En resumen, este ejemplo nos ha proporcionado una ilustración de todas las pautas cizadas: la presentación explícita de las ideas de los estudiantes, el proceso de negociación del status de diferentes ideas, el papel que 
desempeñan los compromisos epistemológicos de los estudiantes y la amplitud e importancia del metaconocimiento.

\section{CONCLUSIONES}

Nuestro objetivo en este artículo ha sido el de caracterizar la enseñanza para un cambio conceptual: es decir, una enseñanza dirigida explícitamente a ayudar a los estudiantes a que experimenten el aprendizaje para un cambio conceptual. AI hacerlo, hemos identificado y discutido unas pautas generales de la enseñanza para un cambio conceptual, hemos considerado varios factores importantes que facilitan la aplicación de esas pautas en las clases normales, $y$ hemos ilustrado estas pautas con ejemplos extraídos de actividades de clase.

La primera pauta se refiere a la necesidad de que las ideas de los estudiantes se hagan explícitas en los debates de la clase. Hay diversas maneras de abordar esta pauta, dependiendo de la forma en que el profesor decida estructurar la clase. Pueden aparecer oportunidades en distintas etapas de la enseñanza de un tema. El primer ejemplo mostraba cómo la exposición de ideas puede tener lugar individualmente o en grupos. El segundo ejemplo demostraba de forma convincente que esta pauta puede abordarse también en el contex to de la enseñanza de estrategias cuyo primer propósito puede no ser la exposición sino, digamos, la construcción de explicaciones sobre un fenómeno.

La siguiente pauta identifica la necesidad de que el status de las distintas ideas constituya una parte explícita del debate en la clase. En este proceso, los estudiantes (iy los profesores!) probablemente se darán cuenta de que habrá cambios en el status que conceden a las diferentes ideas, bajando algunos y subiendo otros. El segundo ejemplo mostraba a varios estudiantes presentando sugerencias que apuntaban directamente al status de la explicación de Don.

La tercera pauta se refiere a la necesidad de una consideración explícita, dentro del programa, de las razones para aceptar o rechazar ideas. Esta patuta es un corolario de la anterior, puesto que el proceso de negoctación del status incluye la consideración de las bases a favor y en contra de la justificación de una idea. Esta pauta, ilustrada más arriba de diferentes maneras, garantiza no sólo que se utilizan estos criterios sino que los alumnos se centran en ellos. Uno de los objetivos del aprendizaje de la Hermana Gertrude consiste en plantear la siguiente pregunta: «iSon consistentes tus ideas?» En el primer ejemplo que hemos presentado, ella presionaba constantemente a los alumnos para que determinaran por qué respondían de la forma en que lo hacían. En el segundo ejemplo, Don utilizaba su concepto de consistencia al argumentar su explicación acerca del descenso del paracáídas.

La úftima pauta identifica la naturaleza metacognitiva de la consideración del status. Ésta, naturalmente, no es independiente de las dos anteriores, pero se ha incluido aquí puesto que ofrece otra manera significativa de entender to que ocurre cuando las personas experimentan el aprendizaje del cambio conceptual. Este punto viene ilustrado en el primer ejemplo, aquél en el que los estudiantes discutian acerca de las varias formulaciones de la inteligibilidad extrádas de la literatura; y en el segundo ejemplo, aquél en el que se presentaba a la clase, para su escrutinio, la explicación de Don.

El ambiente de la clase es un factor esencial en cuanto al grado de observación de estas pautas. En los ejemplos, hemos considerado que tanto el profesor como los alumnos desempeñan distintos papeles que contribuyen a este ambiente de respeto hacia las ideas de los demás, de voluntad de emprender una discusión seria durante amplios períodos de tjempo, y de aceptación de los objetivos de la clase. Creemos que la mayoría de los ambientes en las clases no soportaría la intensidad del escrutinio crítico de las ideas observadas: ni los profesores ni los alumnos están, habitualmente, dispuestos a conceder el tiempo o el respeto a ideas divergentes que parece ser necesario para que tenga lugar este tipo de debate.

Si bien nosotros creemos que las pautas y factores que acabamos de discutir son característicos de la enseñanza para un cambio conceptual, no pretendemos que sean suficientes. Existen aspectos, no discutidos aquí, que la enseñanza para un cambio conceptual comparte con otras formas de enseñanza. Y también hay que señalar que hemos presentado una descripción incompleta del papel del profesor. Los ejemplos señalan varias de las cosas que la Hermana Getrude hizo, pero no deben considerarse que describen todo to que ella hizo. Además, las propias pautas no aparecen suficientemente detalladas: ponerlas en práctica requiere el desarrollo de muchas actividades diferentes relacionadas con el tema considerado y con las ideas que los alumnos aportan por sí mismos.

Cada una de estas pautas y cada uno de estos factores, tomados aisladamente, pueden no parecer muy distintos a las formas habituales de enseñar. Después de todo, los profesores que se han dedicado durante años a la enseñanza de las ciencias han desarrollado actividades y han utilizado estrategias provechosas para los estudiantes y han facilitado en gran manera el aprendizaje de las ciencias: sin duda, la enseñanza para un cambio conceptual ha aprovechado esta enorme base de experiencia. Algunos profesores pueden creer que estamos simplemente dando nombres a métodos habituales que ellos ya han conocido o utilizado y, por tanto, ¿por qué darle más vueltas al asunto? Si bien puede que sea así (hemos tenido este tipo de reacciones en alguna ocasión), nosotros creemos que estas pautas y estos factores combinados entre sí representan un cambio en los métodos actuales. Además, se trata de un cambio que no es, ni mucho menos, incremental. En comparación con la mayoría de las enseñanzas, la enseñanza para un cambio conceptual es una iniciativa radicalmente distinta.

Queda aún una última pregunta: ¿Por qué enseñar para un cambio conceptual? ¿Por qué hacer un esfuerzo para 
Ilevar a cabo la extensa transformación que ello implica? Un tipo de respuesta sería negativo y se derivaría de la insatisfacción con el status quo. Viendo a tantos estudiantes que dejan las clases de ciencias con poco más que un barniz de vocabulario vacío, cxiste una inmensa motivación para encontrar una vía mejor. La enseñanza para un cambio conceptual es una más entre un abanico de diferentes posibilidades. Un segundo tipo de respuesta sería positivo e iría encaminada a conseguir resultados descables. Los ejemplos presentados en las páginas

\section{NOTA}

Ponencia presentada en el IV Congreso Internacional sobre Investigación en la Didáctica de las Ciencias y de las Matemáticas, celebrado en Barcelona los dias 13 al 16 de septiembre de 1993. Ha sido traducida del inglés por J. Tortella.

\section{REFERENCIAS BIBLIOGRÁFICAS}

BEETH, M.E., 1993. Dynamic aspects of Conceptual Change Instruction. Tesis doctoral sin publicar. Universidad de Wisconsin-Madison.

CLIS, 1987. Approaches to teaching the particulate theory of matter. (Universidad de Ieeds: Leeds).

FLAVELL, J. H., 1976. Metacognitive aspects of problem solving, en Resnik, L.B. (ed.), The Nature of Intelligence, pp. 23 I-235. (Lawrence Erlbaum: Hillsdale, $\mathrm{NJ}$ ).

HENNESSEY, M.G., 1991. Analysis of conceptual change and status change in sixth graders' concepts of force and motion. Tesis doctoral sin publicar. Universidad de WisconsinMadison.

HEWSON, P. W., 1981. A conceptual change approach to learning science, European Journal of Science Education, 3 , pp. 383-396

HEWSON, P. W., 1982. A case study of conceptual change in special relativity: The influence of prior knowledge in learning, European Journal of Science Education, 4, pp. 61-78.

HEWSON,P.W. y HEWSON, M.G. A'B., 1988. An appropriate conception of teaching science: $A$ view from studies of science learning, Science Educution, 72, pp. 597-614.

HEWSON, P. W. y THORLEY, N. R., 1989. The conditions of conceptual change in the classroom, International Journal of Srience Education, i1, pp. 541-553. precedentes muestran una calidad en la discusión, una madurez en el método y una profundidad en la comprensión que resultaría impresionante en un nivel de primero de carrera. El hecho de que la discusión la llevaran a cabo estudiantes de quinto grado resulta, a nuestro entender, bastante extraordinario. Si ello es representativo de posibles resultados en el aprendizaje, parece constituir una razón suficiente para enseñar con el objetivo de obtener un cambio conceptual.
MINSTRELL, J., 1982, Explaining the «at rest» condition of an object, Physics Teacher, 20, pp. 10-14.

PALINCSAR, A. S., y RANSOM, K., 1988. From the mystery spot to the thoughtful spot: The instruction of metacognitive strategies, The Reading Teacher, 4I, pp. 784-789

POSNFR, G. I., \$TRIKE, K. A., HEWSON, P. W., y GERT7,OG, W. A. 1982. Accommodation of a scientific conception: Toward a theory of conceptual change, Science Education, 66, pp. $211-227$.

SCOTT, P. H., ASOKO, H. M., y DRIVER, R. H., 1992. Teaching for a conceptual change: A review of strategies, en Duit, R., Goldberg, F. y Niedderer, H. (eds.), Research in Physics Learning: Theoretical Issites and Empirical Studies. pp. 310-329. (IPN: Kiel).

STRIKE, K. A., y POSNER, G. J., 1985. A conceptual change view of learting and understanding, en West, L. H. T. y Pines, A. L. (eds.), Cognitive Siructure and Conceptual Change. (Academic Press: Orlando, Florida).

STRIKE, K. A., y POSNER, G. I, 1992, A revisionist theory of conceptual change, en Duschi, R.A. y Hamilton, R.J. (cds.), Philosophy of science, cognitive psychology, and educational theory and practice, pp. 147.176. (State University of New York Press: Nueva York)

THORLEY, N. R., 1990. The role of the conceptual change model in the interpretation of classroom interactions. 'Tesis doctoral sin publicar. Universidad de Wisconsin-Madison. 